\title{
Beiträge zur Kenntniss des Faserverlaufs im Rückenmark.
}

\author{
Von
}

\section{Dr. P. Schiefferdecker.}

Assistenten am physiulogischen Institut der Universität Strassburg.

Hierzu Tafel XXXII, XXXIII und XXXIV.

Mit Untersuchungen über den Verlauf der Nervenfasern im Rückenmark der höheren Wirbelthiere beschäftigt, gelang es mir vor Kurzem einige Präparate herzustellen, welche mich durch die Klarheit, mit welcher sie den Faserlauf erkennen liessen, überraschten. Bei der Dunkelheit, die auf diesem Gebiete der mikroskopischen Anatomie noch immer herrscht und bei der Wichtigkeit, welche auch noch so geringe Aufschlüsse über den Bau der Centralorgane für die Wissenschaft haben, schien es mir gerechtfertigt, diese Präparate durch Zeichnung und Beschreibung zur allgemeinen Kenntniss zu bringen, zumal auch Herr Prof. Waldeyer, welcher die Güte hatte, dieselben der Durchsicht und Prüfung zu unterwerfen, mir zur Veröffentlichung rieth. Hierbei mag es mir gestattet sein zugleich zu bemerken, dass ich diese kurze Mittheilung nur für eine vorläufige halte, da ich hoffe, dass es mir möglich sein wird, in nächster Zeit eine Monographie über den Bau des Rückenmarks zu veröffentlichen.

Ohne näher auf die ja so reichhaltige Literatur über den vorliegenden Gegenstand einzugehen, will ich mich sofort, indem ich mich nur an Thatsachen halte, zur Beschreibung der Präparate und der Methode, nach der sie gefertigt wurden, wenden.

Die betreffenden Präparate, sowohl Längs- als Querschnitte 
gehören sämmtlich dem Rückenmarke eines und desselben Hundes an und zwar der Partie der Lendenanschwellung, welche den Wurzeln des I. und II. Sacralnervenpaares ihreu Ursprung giebt. Dieser Umstand gewährt uns den Vortheil, die einzelnen Schnitte nicht nur in Bezug auf die Art und Weise des Faserverlaufs, sondern auch in allen einzelnen Grössenverhältnissen unter einander ziemlich genau vergleichen zu können.

Die Methode der Anfertigung war folgende: das Rückenmark, dem frisch getödteten Thiere entnommen und von seinen Hüllen befreit, wurde auf etwa 4 Wochen in Müller'sche Flüssigkeit gelegt, sodann etwa 24 Stunden lang ausgewässert und in Alkohol aufbewahrt. Die Schnitte wurden, um grössere Feinheit und Gleichmässigkeit zu erzielen, mittelst eines kleinen Mikrotoms angefertigt; dann wurden sie in destillirtem Wasser 1-2 Tage lang gründlich ausgewaschen, darauf in Lösungen von Palladiumchlorür (die Querschnitte) und Goldchlorid (die Längsschnitte) eingelegt; nachdem sie die gewünschte Färbung erhalten hatten, wurden sie wiederum mit Wasser tüchtig abgespült, dann in absoluten Alkohol, darauf zur Aufhellung in Nelkenöl und endlich zur Aufbewahrung in Canadabalsam gelegt.

Von dem Palladiumchlorür, welches ich zur Darstellung des Verlaufs der langen geraden Fasern im Rückenmarke auf das Beste empfehlen kann, wurde eine Lösung von 1 : 10000 angewendet, in der die Präparate so lange blieben, bis sie diffus hellbraun gefärbt waren, wozu meist 3-5 Stunden ausreichten, sodann wurden sie einfach in Wasser ausgespült.

Von Goldchlorid, das sich im Gegensatz zum vorigen besonders zur Darstellung der feinen Nervennetze eignet, fand ich ebenfalls Lösungen von $1: 5000$ bis $1: 10000$ am geeignetsten. Nach 13 Stunden waren die Präparate darin ganz schwach violett gefärbt; sie wurden sodann in Wasser abgespült, und auf 24 Stunden in eine $1 / 2-1 \%$ Essigsäure gelegt, darin wiederum abgespült und, wie oben angegeben, behandelt.

Beide Färbungsmittel besitzen wie bekannt die unangenehme Eigenschaft, nicht constant $z u$ färben, und die Präparate nachdunkeln zu lassen, so dass letztere mit der Zeit mehr oder weniger unbrauchbar werden, ein Fall, der z. B. bei den von mir mit Goldchlorid behandelten Längsschnitten zur Zeit, da ich dieses schreibe, bereits eingetreten ist. 
Gehen wir nun zu der Betrachtung der so gefertigten Präparate über.

Die besten Uebersichtsbilder, mit Hülfe deren man die Details dann gut versteht, gewähren uns hier die mit Palladiumchlorür behandelten Querschnitte, von denen ich einen, welcher alles Wesentliche zeigt, abgebildet habe (Fig. $1 \mathrm{a}, \mathrm{b}$ ). Die Zeichnungen sind, mit Ausnahme von zweien, alle von mir selbst gemacht und in allen Einzelheiten des Faserverlaufs so naturgetreu als möglich, soweit sich dieses eben mit Hülfe einer Oberhäuser'schen Zeichenkammer machen liess. Die Feinheiten, welche uns stärkere Objective, z. B. Immersionssysteme auf solchen Präparaten zeigen, sind ja überhaupt nur durch die Photographie, deren Anwendung mir nicht möglich war, darstellbar.

Das Präparat stammte aus der Gegend des Ursprungs des ersten Sacralnervenpaares. Die weisse Substanz ist bei der Zeichnung nur angedeutet, von der grauen sind nur die nervösen Bestandtheile gezeichnet.

Die weisse Substanz, welche in dieser Gegend des Rückenmarks bereits stark gegen die graue zurücktritt, zeigt sich vielfach durchschnitten und zerklüftet von Bindegewebssepten, in denen die Gefässe verlaufen, und welche auch den Nervenfasern oft als Strassen dienen, auf denen sie von der weissen zur grauen Substanz hingelangen. Bei $\mathrm{V}$ haben wir die Vorderstränge bis zu den bei VW durchtretenden vorderen Wurzeln, von da bis zu den bei $\mathrm{HV}$ eintretenden hintern Wurzeln bei S die Seitenstränge, und von hier bis zur Fissura poster. die Hinterstränge $\mathrm{H}$. Die schmale lange Fissura post. ist fast ganz mit Bindegewebe ausgefüllt, verfolgt man sie, so kommt man an die schmale Commis. post., dann auf den hier von hinten nach vorn einen schmalen Spalt bildenden Canal. centr. mit seiner Epithelauskleidung und seiner Umgebung von Bindegewebe. Vor diesem liegt dann die Commissura anterior ${ }^{1}$ ), jene grosse Brücke, welche hauptsächlich den Verkehr zwischen den beiden Rückenmarkshälften vermittelt, zugleich als hintere Grenze der Fiss. ant., welche breiter

1) Ich nenne, um Verwechselungen zu vermeiden, Commiss. anterior den Complex aller jener transveralen Faserzüge, welche vor dem Canal. centr. von einer Seite des Rückenmarks zur andern verlaufen, gleichgültig, ob die Fagern markhaltig sind oder nicht nnd ob sie sich kreuzen oder nicht; ebenso ist die Benennung Commiss. posterior aufzufassen.

M. Schultze, Archip f. mikrosk. Anatomie. Bd. 10. 
und kürzer als die Fiss. post. und nur zum kleinen Theile mit Bindegewebe erfüllt die beiden Vorderstränge von einander trennt.

Was die graue Substanz anbetrifft, so zeigt sich auf den ersten Blick, wie unzureichend zur Beschreibung speciellerer Verhältnisse derselben die Eintheilung in Vorder- und Hinterhörner ist, da absolut keine Grenze zwischen beiden existirt. Ich ziehe es daher zum Zwecke der Beschreibung vor, die graue Substanz durch eine künstliche Grenze, welche ich mir von den Proc. reticulares (Pr.) nach dem hintern Ende des Spaltes des Can. centr. gezogen denke, in zwei verschieden grosse Theile zu theilen, eine Eintheilung, die übrigens auch darin ibren Grund findet, dass für diesen Theil des Rückenmarks wenigstens diese beiden Abtheilungen für den Faserverlauf und somit auch für die Function von verschiedener Bedeutung sind. Der Rand der weissen Substanz nach der grauen hin ist vielfach zerklüftet, und sieht auf dem Querschnitt wie eine von zahlreichen Fiorden zerschnittene Küste aus, mit vielen vorliegenden Inseln und Inselchen in dem Meere der grauen Substanz. Diese Bildung ist hauptsächlich den Seitensträngen, dem an die Comm. ant. grenzenden Theile der Vorderstränge und der vor den eintretenden hintern Wurzeln liegenden Partie der weissen Substanz eigenthümlich, welche gewissermassen zwischen Seiten- und Hintersträngen liegt.

In dem nach unserer Eintheilung vorderen Abschnitte der grauen Substanz nun, der etwa die Gestalt eines unregelmässigen Fünfecks mit abgerundeten Ecken hat und den hinteren an Grösse ungefähr viermal übertrifft, finden wir drei Gruppen grosser Ganglienzellen, die sowohl an Zahl der in ihnen enthaltenen Zellen, wie an Grösse und Art und Weise der Anordnung derselben unter einander verschieden zu sein pflegen. Diejenige Gruppe, welche die meisten und grössten Zellen enthält, liegt in jener tiefen und umfangreichen Bucht, welche die Seitenstränge in der Höhe des Can. centr. bilden, wir wollen sie einfach als die seitliche Gruppe bezeichnen. Die Zahl der Zellen, welche man auf den verschiedenen Querschnitten zu Gesicht bekommt, ist sehr verschieden, ich habe sie zwischen 8 und 25 schwankend gefunden, denn die Säule, welche diese Gangliengruppe am Rückenmark bildet, zeigt, wie wir später bei den Längsschnitten des Genaueren sehen werden, fortdauernd auf einander folgende Einschnürungen und Ausbauchungen. Der Durchmesser der Zellen ist durchschnittlich 0,07 Mm. Was die Lagerung derselben zu einander anbetrifft, so habe ich hier keine bestimmte Anordnung entdecken 
können, sie liegen eben meist zu einem Haufen vereint ordnungslos mehr oder weniger eng aneinander. Die Gruppe ragt, obwohl ihre äussersten Zellen meist der weissen Substanz dicht anliegen, doch weit in die graue Substanz hinein. Die zweite Ansammlung von Nervenzellen, die vor der e, findet sich am Rande der weissen Vorderstränge an denjenigen Stellen, an denen die vorderen Wurzeln durchtreten. Die Grösse der Zellen ist geringer als die der vorigen, der Durchmesser beträgt im Durchschnitt $0,05 \mathrm{Mm}$. Ihre Zahl auf dem Querschnitte ist ebenfalls schwankend, sie betrug zwischen 6 und und 18. Die Zellen sind hier zu lauter kleinen Häufchen von 2-6 abgetheilt welche entweder gerade dem Austrittspunkte einer vorderen Wurzel gegenüber, oder zwischen zweien solcher in der Mitte liegen.

Die dritte Gruppe von Ganglienzellen endlich, die mittlere hintere, liegt etwa an der Grenze des ersten und zweiten Dritttheils unserer imaginären Linie vom Can. centr. aus gerechnet und befindet sich entweder noch vor dieser Linie, ihr dann dicht anliegend, oder wird bereits von ihr geschnitten. Die Zahl der Zellen schwankt hier auf den Querschnitten zwischen 4 und 8, ihre Grösse ist der der Zellen der vorigen Gruppe etwa gleich, sie liegen meist in zwei mehr oder weniger weit von einander getrennte Häufchen gesondert. Diese Gruppe ist wohl als das Analogon der Clarke'schen Säulen des Dorsalmarks im Lendenmark aufzufassen.

Ausserdem giebt es natürlich hin und wieder auch einzelne abgetrennt liegende Ganglienzellen, doch bilden sie die entschiedenen Ausnahmen.

Die kleinen Ganglienzellen der Hinterhörner habe ich auf den Zeichnungen nicht berücksichtigt, da sie bei der angewandten schwachen Vergrösserung auf den Präparaten nicht zu erkennen waren.

Bei Betrachtung des Faserverlaufs müssen wir zunächst den in der grauen und den in der weissen Substanz unterscheiden.

Was den Verlauf der Fasern in der grauen Substanz anbetrifft, so zeigte sich derselbe auch schon, wenn man nur, wie ich es gethan habe, die gröberen Züge der Nervenfasern berücksichtigte ${ }^{1}$ ), ganz ausserordentlich complicirt.

Wir können zunächst 5 Hauptarten von Fasern je nach ihrem Ausgangs- und Zielpunkte unterscheiden:

1) Wenigstens auf den Querschnitten, auf den Längssehnitten sind auch die feinen Nervenbündel angegeben. 
1) Fasern, welche von einem Punkte der Peripherie zu Ganglienzellen laufen.

2) Fasern, welche von einer Ganglienzellengruppe $\mathrm{zu}$ einer zweiten verlaufen.

3) Fasern, welche von einem Punkte der Peripherie nach einer Commissur hin verlaufen.

Hier sind dann die Ganglienzellengruppen der centralen Commissur gegenüber ebenfalls als Peripherie aufgefasst.

4) Fasern, welche in der grauen Substanz senkrecht zur Querschnittsłäche als Verbindungsfasern böherer und tieferer Partieen verlaufen.

5) Fasern, welche in derselben Hälfte des Rückenmarks bleibend direct von einem Theile der weissen Substanz zu einem anderen binlaufen.

Ich habe hier bei Bezeichnung des Laufes der Nervenfasern absichtlich die Worte Ausgangs- und Zielpunkt gebraucht, nicht Anfang und Ende, da ich dieselben nur zum Zwecke der Beschreibung benutzen wollte, ohne weiter auf die Frage einzugehen, wo Fasern entspringen oder endigen, oder nach welcher Richtung die Leitung in ihnen erfolgt.

Eine allgemeine Eigenschaft der in der grauen Substanz verlaufenden Faserzüge ist die, dass sie entweder in enggeschlossenen Bündeln austreten und sich dann allmählig immer feiner und feiner nach allen Richtungen hin pinselförmig ausbreiten, oder dass sich eine Menge dünner Fasern alluählig zu einem Bündel sammelt. Hieraus allein folgt nun scbon, dass man auf Querschnitten verhältnissmässig selten Fa-ern ihrer ganzen Länge nach verfolgen kann, doch schadet dieses nicht so viel, da man meist Bündel antrifft, welche schräg von unten herauf- oder von oben herabsteigend den gleichen Weg einschlagen, und als gute Wegweiser für den ferneren Verlauf betrachtet werden können. Dass diese Behauptung richtig ist, zeigt am besten die Betrachtung der Längsschnitte.

Gehen wir nun zur speciellen Beschreibung des Faserverlaufs selbst über.

Aus dem ganzen inneren Umfange der weissen Substanz sehen wir unzählige gröbere und feinere Nervenbündel austreten und nach den verschiedensten Richtungen sich verbreiten. Am längsten bleiben diejenigen Fasern in Bündeln zusammen und verfolgen also denselben Weg, welche aus den Hintersträngen kommen; am schnellsten fahren 
sie nach allen Richtungen auseinander bei den durchtretenden vorderen Wurzeln. Die Seitenstränge stehen zwischen beiden, sie bilden gewissermassen den allmähligen Uebergang zwischen den beiden Extremen. Der Grund dieses Unterschiedes liegt in der Länge des Weges, den die betreffenden Fasern von ibren Austrittspunkten bis zu ihren Zielpunkten zurückzulegen haben: je kürzer dieser Weg ist, um so schneller werden sich die verschiedenen Fasern eines Bündels voneinander trennen, um ihren verschiedenen Zielpunkten zuzulaufen, wobei dann die mannichfachsten Kreuzungen verschiedener Faserzüge zu Stande kommen.

Unsere erste Abtheilung enthielt diejenigen Fasern, welche von der Peripherie zu Ganglienzellengruppen laufen.

Von wo überall her laufen nun Fasern zu den drei verschiedenen Gruppen?

Am meisten bevorzugt ist in dieser Beziehung wieder die seitliche Gruppe; wie sie die meisten und grössten Zellen enthält, so erhält sie auch die meisten und verschiedenartigsten Faserzüge.

Erstens kann man deutlich dahin verfolgen grosse Faserzüge aus den vorderen durchtretenden Wurzeln (Fig. 1 a, b). Die medialen, der Fiss. ant. anliegenden Partieen der Vorderstränge scheinen sich nicht oder wenigstens nur mit sehr geringen Faserzügen zu betheiligen.

Zweitens treten zu dieser Gruppe eine Menge von Fasern der Seitenstränge und zwar aus allen Gegenden derselben.

Drittens ziehen ohne Zweifel starke Bündel aus den Hintersträngen dorthin, und zwar hauptsächlich aus den vorderen schmalen medialen Theilen derselben (Fig. 1 a).

Alle diese Faserbündel nun, welche an jenem Orte zusammenkommen, lösen sich fein pinselförmig auf, ver\#lechten sich dadurch, dass sie nach allen Seiten hin zwischen den Ganglienzellen hindurch und um dieselben herumlaufen, auf das innigste und mannichfaltigste (oft laufen sie fast kreisförmig um die Zellen und Gruppen herum), und bilden so allmählig ein feines Netzwerk (wie das besonders bei den Längsschnitten deutlich werden wird), in das ohne Zweifel auch die Fortsätze der Ganglienzellen eintreten, wenn mir dieses letztere auch sichbar zu machen bisher noch nicht gelungen ist.

$\mathrm{Zu}$ der zweiten Ganglienzellengruppe, welche den Vordersträngen anliegt, begeben sich nicht so viel Fasern. Es sind folgende: 
Erstens und vor allen Dingen ein Theil der Züge der durchtretenden vorderen Wurzeln. Iich erwähnte oben, dass die Ganglienzellen in dieser Gruppe zu kleinen Häufchen geordnet seien, welche theils den Durchtrittspunkten der Wurzeln gegenüber, theils zwischen denselben gelagert seien. Es tritt in Folge dessen auch die Hauptmenge der Fasern eines zutretenden Bündels zu der zunächst liegenden Gruppe hin und verzweigt sich zunächst in ihr, doch treten auch Fasern zu den weiter liegenden Häufchen, und ausserdem stehen alle diese Gruppen, diese kleinen Ganglien oder Ceutren, wie man sie nennen könnte, noch durch feine aus dem zwischen den Zellen sich bildenden Nervennetze entspringende Fäserchen untereinander in engerer Verbindung. Auch diese Verhältnisse zeigen uns die Längsschnitte besonders klar. Bis zur Umbiegungsstelle der Vorderstränge nach der Fiss. ant. hin reicht das Gebiet, aus dem die $z \mathfrak{u}$ den Zellen laufenden Fasern kommen, die medialen Partieen scheinen keine zu entsenden.

Ferner treten von den Seitensträngen aus eine Menge von Faserzügen zu der vorderen Zellengruppe hin. Dieselben gehören sämmtlich der vorderen Hälfte derselben an, die hintersten treten zur Seite oder dicht hinter der seitlichen Nervenzellengruppe aus und laufen um diese im Bogen herum, sowohl lateral als medial, um nach vorne zu gelangen.

Endlich laufen auch von den Hintersträngen und zwar, wie es scheint, gerade von den medialen Partieen derselben dünne Faserzüge za den vorderen Ganglien hin. Die Querschnittsbilder liessen eine solche Verbindung bereits vermuthen, doch waren sie nicht beweisend; der in der Fig. 5 dargestellte Längsschnitt indessen, den wir später noch genauer betrachten, wollen, liess an Klarheit kaum etwas zu wünschen übrig. Es sind dieses jedenfalls Faserzüge, die anfangs mit den zu der hinteren mittleren Ganglienzellengruppe gehenden zusammen oder doch in der Nähe derselben verlaufen, später aber dann lateral an dieser Gruppe vorbei nach vorne ziehen, und bei diesem weiteren Verlaufe leicht mit den von der hinteren mittleren zu der vorderen Ganglienzellengruppe verlaufenden Verbindungsfasern verwechselt werden können.

Die letzte und kleinste der drei Nervenzellengruppen endlich, die hintere mittlere enthält Fasern aus zwei Theilen der weissen Substanz : aus den gesammten Hintersträngen und der hintereu Hälfte der Seitenstränge, namentlich für die ersteren scheint sie von Wich- 
tigkeit zu sein, wenn man diesen Schluss aus der Menge der hinzulaufenden Fasern ziehen darf.

Unsere $\mathrm{z}$ weite A b theil ung umfasste diejenigen Fasern, welche von einer Ganglienzellengruppe zu einer zweiten hinziehen, also gewissermassen Commissuren zwischen denselben bilden. Wir haben drei Haufen von Zellen, die alle untereinander verbunden sind, also auch drei solcher Faserzüge. Der zwischen der seitlichen und vorderen Gruppe ist auf beiden abgebildeten Rüchenmarkshälften gut zu erkennen, der zwischen der seitlichen und der hinteren ebenfalls auf beiden, der zwischen der vorderen und der hinteren nur auf Fig. $1 \mathrm{~b}$ deutlich sichtbar.

Wir kommen somit zu der d ritten Fas era rt, zu denen, welche von einem Punkte der Peripherie (weisse Substanz oder Ganglienzellengruppen) aus zu einer der zwei, die beiden Seitenhälften des Rückenmarks verbindenden Commissuren sich begeben. Beginnen wir mit der Comm. ant. Man kann sich hier recht kurz fassen und wird kaum etwas unrichtiges behaupten, wenn man sagt: es giebt kaum einen Punkt auf der ganzen Peripherie der weissen Substanz, es giebt keine Ganglienzellengruppe, von wo aus nicht Fasern in die vordere Commissur einträten. Mächtige dunkle Faserbündel, aus allen Partieen der Hinterstränge mit Fasern versorgt, steigen dicht neben dem Can. centr., die hintere Zellengruppe von beiden Seiten umfassend, zu der vorderen Commissur hinauf (Fig. 1 a und besonders gut $1 \mathrm{~b}$ ). Noch grösser ist die Masse der aus den Seitensträngen und zwar besonders wieder aus dem hinteren Theile derselben zu jener Verbindungsbrücke hinziehenden Bündel, und auch die Vorderstränge liefern aus allen ihren Theilen ein nicht unbedeutendes Contingent. Endlich ziehen von jeder der Gangliengruppen noch mehr oder weniger starke Bündel dorthin. Der grösste Theil dieser Faserzüge kreuzt sich bekanntlich in der,Commissur, um dann der Hauptmenge nach wenigstens wohl in die medialen Partieen der Vorderstränge einzubiegen, ein Theil indessen und zwar der hintere, dem Can. centr. zunächst liegende, geht ohne Kreuzung als einfache Verbindungsfasern von einer Seite zur andern. Die hier beschriebenen Präparate zeigten diese Faserzüge nicht deutlich, sondern enthielten nur Andeutungen derselben, auf Querschnitten aus dem Halsmarke des Kalbes indess, welche mit Goldchlorid behandelt waren, habe ich diese Verbindungszüge mit grosser Deutlichkeit gesehen. Dass die in der vorderen Commissur sich kreuzenden Fasern nicht 
in der Horizontalebene verlaufen, sondern mit dieser oft einen recht bedeutenden Winkel bilden ist ja eine längst bekannte und auf Frontalschnitten leicht zu constatirende Thatsache.

Viel weniger bedeutend sind die Faserzüge, welche die hintere Commissur passiren. Dieselben gehen einmal von den medialen Partieen der Hinterstränge und zweitens von der binteren mittleren Ganglienzellengruppe aus (Fig. $1 \mathrm{a}, \mathrm{b}$ ) und laufen einfach parallel und ohne sich zu kreuzen auf die andere Seite hinüber.

Was die beiden letzten Faserarten endlich anbetrifft, diejenigen, welche senkrecht zum Querschnitt verlaufen und die, welche zwei verschiedene Theile der weissen Substanz derselben Seite direct verbinden, so besprechen wir dieselben besser bei der Betrachtung der Längsschnitte.

Noch möchte ich auf den Verlauf einiger Faserzüge besonders aufmerksain machen, die bisher nur im Allgemeinen erwähnt sind und speciell dem hinteren Abschnitte angehören.

Erstens finden wir dicht an dem Rande der Seitenstränge hinter den Proc. reticul. einen starken Faserzug, welcher nach hinten zu in einem Bogen bis zu der ebenfalls netzartig gebildeten Partie der weissen Substanz sich hinzieht, welche dicht vor dem Eintritt der hinteren Wurzeln liegt. In dieser Gegend treffen die Faserbündel dann, indem sie sich theilweise kreuzen und durcheinander laufen, mit jenen weniger mächtigen Faserzügen zusammen, welche von hier am Rande der Hinterstränge bogenförmig weiter verlaufen, und allmählig in verschiedenen grösseren und kleineren Bündeln mit anderen Fasern zusammen die Subst. gelat. Rolandi nach vorn durchziehen. Die an den Seitensträngen sich hinziehenden Faserbündel nehmen, wie es scheint, ihren Ursprung zum grössten Theil aus den Strängen dieser hinteren netzförmigen Substanz, zum sehr viel kleineren Theile aus den apliegenden Partieen der Seitenstränge, weiterhin erhalten sie dann noch Fasern aus den Strängen der Proc. reticul. und laufen alsdann sich theilend theils zu der hinteren mittleren, theils $\mathrm{zu}$ der grossen seitlichen Ganglienzellengruppe.

$\mathrm{Z}$ weiten s sehen wir, dass aus den weiter in die graue Substanz vorgeschobenen Strängen der Proc. reticul. Faserbündel entspringen, welche nach den verschiedensten Richtungen verlaufen. Einmal sind es nämlich solche, welche analog dem Verhalten der übrigen Theile der Seitenstränge nach vorn und aussen zu der grossen seitlichen Ganglienzellengruppe und nach vorn und innen $\mathrm{zu}$ der kleinen mitt- 
leren hinziehen, dann aber finden wir drittens auch noch constant einige besonders von den hinteren dieser vielen kleinen Stränge abgehende Bündel, welche zuerst gerade nach innen verlaufen und dann sich pinselförmig auflösend den aus den Hintersträngen kommenden Faserzügen entgegen laufen und sie unter verschiedenen Winkeln kreuzen. Wo eigentlich der Zielpunkt dieser Fasern ist, war mir bisher noch nicht möglich nachzuweisen, wahrscheinlich werden sie aber sich immer feiner zertheilend mit einem in diesen Gegenden von den aus den Hintersträngen austretenden Fasern gebildeten feinen Nervennetze, dessen Existenz in einer nur wenig weiter nach vorn gelegenen Region mir auf Längsschnitten nachzuweisen gelang, in Verbindung treten, sich mit den andern Fasern verflechten und weiterhin mit ihnen zusammen verlaufen.

D rit te $\mathrm{n}$ s endlich möchte ich noch auf die ungemein zahlreichen Kreuzungen aufmerksam machen, welche die aus den Hintersträngen entspringenden Fasern gleich nach ihrem Austritt dicht an dem Rande der weissen Substanz untereinander ausführen und welche uns alle Abbilduvgen besonders gut aber Fig. VII (die mit stärkerer Vergrösserung, Obj. 5, gezeichnete hintere Partie von I b) zeigen. Es wird hierdurch bewirkt, dass in den ihren Zielpunkten nach ja durchaus nicht einander gleichwerthigen Faserbündeln, welche die Subst. gelat. Rol. nach vorne durchsetzen, sich Fasern befinden, welche aus den verschiedensten Theilen der Hinterstränge entspringen. Bei Betrachtung des Faserverlaufs in der weissen Substanz werden wir hierauf noch einmal zurückkommmen.

Durch diese vielen grossen sich auflösenden und nach den verschiedensten Richtungen durch- und übereinander verlaufenden Faserzüge, welche ich mich wenigstens ihrer gröbsten Anordnung nach zù beschreiben und in ihrem Verlaufe klar zu legen bemüht habe, entsteht also jenes fabelhafte Fasergewirr, welches uns das Studium des feineren Baus des Rückenmarks und die Feststellung der Bahnen so erschwert. Ein Blick auf Präparat oder Zeichnung hilft hier natïrlich mehr als alle Beschreibung, deren Aufgabe wiederum es ist, auf die Einzelheiten aufmerksam zu machen und die Gesetzmässigkeit der Anordnung hervorzuheben.

Von den vielen Längsschnitten, welche ich zum Zwecke der Untersuchung angefertigt hatte, waren nur wenige mittelst der Goldchloridbehandlung so glücklich gelungen, dass sie einen klaren Einblick in den Faserverlauf gewährten; von diesen wenigen sind einige 
als Zeichnungen dieser Arbeit beigegeben worden und sollen jetzt ihre Besprechung finden. Diese Zeichnungen sind auf die nämliche Weise wie die der Querschnitte angefertigt worden (Oberhäuser'sche Zeichenkammer und 0 bj. 3), wenigstens was die allgemeinen Umrisse und den Verlauf der gröberen Faserzüge anbetrifft, und hierin sind sie also ganz genau, die Detailzeichnung ist wenigstens eine annähernd genaue Copie des Originals. Auch hier ist nur auf die nervösen Elemente Rücksicht genommen. Was die Richtung der Schnitte betrifft, so ist dieselbe auf Fig. $1 \mathrm{~b}$ durch je zwei in der Fortsetzung derjenigen Linie gelegene Punkte, in welcher der Längsschnitt den Querschnitt treffen würde, angegeben.

Fig. 2 zeigt uns einen Schnitt, der von dem hinteren Theile der Seitenstränge nach dem vorderen Theile des Canal. centr. gelegt ist, also etwa frontal. Wir sehen auf demselben ganz links bei a einen Fetzen des das Rückenmark zunächst umhüllenden Bindegewebes, dann folgt ein breiter Streifen weisser Substanz der Seitenstränge bei $b$ mit meist parallel laufenden Nervenfasern, nur am Rande lösen sich einige Bündel zeitweilig ab, um ein Ende durch die graue Substanz zu verlaufen und dann wieder zu der weissen zurückzukehren, wobei dann ein Uebereinanderlaufen vielfach unvermeidlich wird. Solche Bündel bilden auf dem Querschnitte die an dem Rande der Seitenstränge liegenden Inselchen in der grauen Substanz. Aus der weissen Substanz sieht man bei c eine Reihe von mässig dicken, einander ziemlich parallel und zu den Fasern der weissen Substanz an der Austrittsstelle etwa senkrecht verlaufende Faserbündel austreten, deren Anfänge in der weissen Substanz man indessen verschieden weit in dieselbe hinein, oft bis über die Mitte derselben hinaus verfolgen kann: es sind sämmtlich Fasern, die von oben herab kommend nach der grauen Substanz zu sich umbiegen, und die in den verschiedensten Theilen der weissen Substanz herab. zusteigen scheinen. Nachdem diese Faserbündel ausgetreten sind, beginnen sie bald sich pinselförmig zu zerfasern, und diese Zertheilung wird schliesslich etwa in der Mitte der grauen Substanz so beträchtlich, dass die Fasern der einzelnen Bündel sich mit denen der ein bis zwei nächsten Bündel jederseits auf das innigste verflechten: es findet hier also ein allgemeiner Austausch der Fasern der austretenden Bündel statt. Nachdem dieser Gipfelpunkt der Zertheilung erreicht ist, eilen die einzelnen Fasern sich wieder zu neuen Bündeln zu einen, welche meist breiter sind als die austretenden, da die Fasern nicht 
so dicht aneinander gedrängt in ihnen liegen. Aus eben diesem Grunde erscheinen diese secundären Bündel auch nie so dunkel gefärbt als die primären. Diese zweite Reihe von Nervenbündeln, welche den austretenden also keineswegs entsprechen, da jedes von ihnen Fasern aus 4-5 der ersteren enthält, laufen dann schräg nach vorn und unten, um an dem Can. centr., dessen Bindegewebsumgebung und Epithel wir bei $d$ und e sehen, vorbei nach der Comm. ant. zu ziehen, um sich durch diese auf die andere Seite zu begeben. Sie entsprechen also den grossen Faserzügen, welche wir auf dem Querschnitte in der bezeichneten Gegend so reichlich von den Seitensträngen zu der Comm. ant. hinziehen sehen. Unserer Eintheilung nach würden es Fasern der dritten Abtheilung sein.

Es folgen drei Schnitte, welche mehr oder weniger genau sagittal gelegt sind und die Vorder- uud Seitenstränge betreffen.

Fig. 6 zeigt einen ziemlich genau sagittalen Schnitt durch die weisse Substanz der Seitenstränge, welcher in der Mitte noch ein kleines Stück der grauen Substanz gerade an der Stelle der tiefen Einbuchtung der Seitenstränge nebst einigen der seitlichen Gruppe angehörigen Ganglienzellen mitgenommen hat. Interessant ist hier hauptsächlich der Faserverlauf der weissen Substanz. Während die Aussentheile uns nur parallel laufende Fasern zeigen, sehen wir die mittlere Partie, welche etwa den dritten Theil des Präparats ausmacht, von Faserzügen eingenommen, welche sich fast zopfartig miteinander verflechten. Nach den Seiten hin findet ein ziemlich rascher Uebergang statt. Je näher der grauen Substanz, um so stärker wird die Verflechtung, um so grösser werden die Maschen des Netzwerks, bis endlich noch durch die graue Substanz selbst zahlreiche Bündel von einem Pfeiler der weissen Substanz zum andern hinüberlaufen, so dass das Ganze den Eindruck macht, als ob man von innen, von der grauen Substanz her, versucht hätte die weisse Substanz nach Aussen umzubiegcn und dieselbe nun auf der inneren Seite in Folge dessen auseinander geplatzt wäre, wobei dann, wie das ja bei faserigen Stoffen immer der Fall ist, verschiedene Rissstellen in verschiedenen Höhen entstanden wären, wodurch natürlich schräg von einer Seite zur andern laufende, Faserbündel bedingt wären. Es sind dieses wieder dieselben Bündel, welche wir auf dem vorigen Präparate am Rancle der weissen Sub$\operatorname{stanz}$ vorfanden. Bei a endlich sehen wir wieder einige aus der weissen Substanz austretende Faserzüge, welche in derselben wiederum 
von oben herabgestiegen sind, und sich jetzt $z u$ den Ganglienzellen begeben.

Auf Fig. 4 haben wir eine der Mitte des Rückenmarks näher gelegene Partie vor uns, die Schnittebene bildet mit der Sagittalebene einen Winkel von etwa $25-30^{\circ}$. Ganz rechts bei a finden wir wieder die Fasermasse des Seitenstranges, aus welchem ziemlich genau unter einem rechten Winkel eine sehr bedeutende Menge von Nervenbündeln austreten. Dieselben sind von sehr verschiedener Dicke und es liegen zwischen zwei starken Bündeln immer mehrere schwächere. Alle diese Bündel zerfasern sich piuselförmig, doch ist auch hier wieder ein Unterschied zwischen den dickeren und den dünneren bemerkbar. Die letzteren beginnen ihre Zertheilung sehr bald nach ihrem Austritt und bilden so, sich gegenseitig innig verflechtend ein feines und zierliches Netzwerk, zu welchem die dickeren Bündel während ihres Durchtretens durch dasselbe ebenfalls dünnere Zweige hinsenden. In diesem Nervennetze sehen wir an einigen Stellen dunkle Punkte, von denen eine Menge feiner Ausläufer ausgeht, welche sich in dem Nervennetze verlieren und genau dieselbe Beschaffenheit haben, wie die anderen das Netzwerk bildenden Fasern. Ich halte diese Punkte für den Ausdruck von Quer- oder Schrägschnitten solcher Faserbündel, welche mit der Ebene des Schnittes einen ziemlich bedeutenden Winkel in ihrem Verlaufe bilden (dass solche in grosser Menge vorhanden sind, zeigen ja die Querschnitte); wir sehen also gewissermassen von oben in die pinselförmige Ausbreitung dieser Bündel hinein. Das Nervennetz, welches ich mir übrigens nicht durch wirkliche.Anastomosen, sondern durch Auflösung und Verflechtung der Faserbündel entstanden denke, dient nun zwei verschiedenen $Z$ wecken: einmal nämlich bewirkt es eine allgemeine Vermischung der Fasern aus den verschiedensten Theilen des Seitenstranges durch Zu- und Abtreten derselben von den grossen durchtretenden Bündleln, und zweitens giebt es anderen Nervenbündeln ihren Ursprung, welche vertieal aufsteigen und verschiedene Etagen des Rückenmarks mit einander verbinden, doch erhalten dieselben theilweise wenigstens auch direct durchtretende Fasern. Neue, von den durchtretenden Nervenbündeln ganz unabhängige horizontal verlaufende Bündel scheinen sich meinen Beobachtungen nach aus dem Netze nicht zu bilden, jene weiter nach der Mitte zu plötzlich auftauchenden dickeren Nervenbündel halte ich ebenfalls für solche, welche mit der Schnittebene einen in diesem Falle sehr spitzen Winkel bilden. Die dickeren Bündel also 
laufen, nachdem sie bei dem Durchtritt durch das Netz die mannichfachsten Verbindungen eingegangen sind und nachdem sie vielfach grössere und kleinere Bündel an die vertical anfsteigenden Faserzüge abgegeben haben, sich mehr und mehr verästelnd etwa bis zur Mitte der grauen Substanz noch als ziemlich starke Bündel hin. Einzelne Ganglienzellen, welche der Seitengruppe angehören, werden im Vorbeiziehen umstrıckt und geben wahrscheinlich ihre Ausläufer mit. Von der Mitte an dagegen beginnt die Auflösung der Bündel ausserordentlich rasch vor sich zu gehen, überall sieht man breite Pinsel, von feiuen Fasern sich auf das mannichfachste durchflechten: es findet also zum zweitenmale ein neues und grïndliches Durcheinandermischen der Fasern statt. Dann sammeln sich dieselben wieder zu neuen Bündeln (die den primären also nicht mehr entsprechen), indess nur um sich in oder zwischen die verschiedenen kleinen Häufchen der vorderen Ganglienzellengruppe $z u$ begeben, durch welche sie allem Anscheine nach theilweise wohl direct hindurchtreten (Fasern der fünften Abtheilung), zwischen denen sie zum grösseren Theile aber sich wieder in ein neues und sehr feines, mit den Ausläufern der Zellen sicher in Verbindung stehendes Netz auflösen, um aus diesem wieder zu neuen Bündeln vereinigt auszutreten, und endlich zusammen mit den direct durchgetretenen Bündeln als austretende vordere Wurzeln schräg nach unten die weissen Vorderstränge zu durchsetzen und die weiter nach der Peripherie verlaufenden Nervenstämme zu bilden. Die einzelnen Häufchen der Ganglienzellen der vorderen Gruppe hängen auf dem Längsschnitte übrigens ebenso gut durch feine Faserbündel zusammen wie auf dem Querschnitte, wir können uns also, indem wir uns diese Gangliengruppen körperlich vorstellen, einen Begriff bilden von der collossalen Menge von Verbindungen, welche hier existiren.

Andere Präparate, deren Zeichnungen ich hier nicht beigefügt habe, zeigten in mancher Hinsicht noch schöner die eigenthümliche baumartige Form der dicken aus den Seitensträngen austretenden Bündel mit ihrer so zierlichen Verästelung und dem schön geschwungenen Verlauf der Fasern, ganz im Gegensatze zu den breiten ruthenartigen, hässlich steifen Bündeln der vorderen durchtretenden Wurzeln, und bewiesen zugleich, dass jene senkrecht aufsteigenden Faserzüge durchaus nicht allen Partieen dieses Theiles des Rückenmarks eigen sind.

Fig. 3 ist dadurch sehr interessant, dass sie uns Auflulärung 
über das Verhalten der seitlichen Ganglienzellengruppe gegenüber den austretenden Fasern giebt.

Bei a bemerken wir wieder die Seitenstränge, aus denen ein wahrer Wald von dickeren und dünneren Nervenbündeln austritt, welche wiederum durch das bekannte feine Netzwerk miteinander in Verbindung stehen. Alle diese sich weithin verzweigenden Bündel laufen zu einer Säule grosser Ganglienzellen hin (seitliche Gruppe), welche, wie schon oben erwähnt, bald Einschnürungen bald Anschwellungen zeigt, je nach der Menge der Zellen, um zum Theil zwischen diesen Zellen hindurchzutreten, zum Theil sich in dem sehr feinen dort befindlichen Nervennetze aufzulösen. Hier kommen nun sicher die mannichfaltigsten Verbindungen und Verflechtungen der Fasern mit den Zellen und den Fasern der verschiedenen Bündeln unter sich vor. Aus dem Netzwerke verden dann einmal vertical laufende Bündel gebildet, welche theils verschieden boch gelegene Partieen der Ganglienzellensäule in Verbindung setzen, theils wohl auch später seitlich umbiegende Fasern nach andern Partieen senden mögen (Fasern der vierten Abtheilung bei $\mathrm{x}$ ), und zweitens neue horizontal verlaufende Stämme, welche sich entweder den schon vorhandenen einfach durchtretenden anschliessen, oder selbständig als neue Bündel weiter nach vorn ziehen. Einige von dem grossen Haufen etwas entfernte, zerstreut liegende Ganglienzellen bilden unterwegs noch neue Verknüpfungspunkte, bis endlich wieder jene allgemeine pinselförmige Auflösung und innige Verflechtung untereinander stattindet, aus der auf die ja bei den vorigen Präparaten bereits beschriebene Weise sich endlich mit Hülfe der vorderen Gangliengruppe und eines neuen Netzwerks daselbst die austretenden vorderen Wurzeln bilden. Auch in einer anderen Beziehung ist dieses Präparat noch interessant; durch die breite Fasermasse des Seitenstranges zieht sich ein heller Streifen, welcher anzeigt, dass das Präparat hier um vieles dünner ist als sonst, wie das ja bei einer etwas unsicheren Schnittführung leicht vorkommt. Diese hellere Partie nun setzt uns in den Stand, den Verlauf der Anfänge der austretenden Fasern in der weissen Substanz weit zu verfolgen: auch hier kommen wieder die sämmtlichen Fasern von oben herab, und zwar in den verschiedensten Theilen des Seitenstranges, sowohl den äusseren wie den inneren.

Fig. 5 giebt uns eine Anschauung von der zwischen den Hinterund Vordersträngen bestehenden Faserverbindung. 
Bei a bemerken wir die Parallelfaserzüge des schmalen medialen Theils der weissen Substanz der Hinterstränge noch bekleidet von einem Feizen des die Fiss. post. ausfüllenden Bindegewebes. Zierliche feinere und gröbere Nervenbündel, welche jedoch nicht die Stärke der aus den Seitensträngen austretenclen erreichen (ein Umstand, der sich wohl hinreichend erklärt durch die geringere Dicke der weissen Substanz und die grössere Feinheit der Fasern) ziehen in die graue Substanz hinein. In einer bestimmten Entfernung von dem Rande der weissen Substanz Iösen sich diese Bündel in ein feines Nervennetz auf, welches eine ziemlich bedeutende Breite besitzt. In diesem Netz scheinen sich alle Nervenbündel aufzulösen und, wenn auch einige tief in dasselbe hineintreten, als wenn sie es einfach durchsetzen wollten, so creilt sie ihr Schicksal doch, nur etwas später als die übrigen. Auch in diesem Netz finden wir jene dunklen Punkte mit ihrem Faserkranz wieder, die wir schon auf Präparat 4 zu bemerken Gelegenheit hatten, nur dass sie hier gemäss der geringeren Dicke der Bündel ebenfalls uns kleiner erscheinen. Es treten also auch hier von den Seiten her radial verlaufende Nervenbündel in das Netz ein. Auf diese Zone des Nervennetzes folgt weiterhin d. h. also nach vorn $z u$, eine Menge einander parallel laufender. jedoch vielfach untereinander durch schräg verlaufende Aeste sich verbindender mässig dicker Faserbündel (bei $\mathrm{x}$ ), welche senkrecht aufsteigen. Nur an einigen Stellen zieht sich das Nervennetz bis zwischen dieselben hinein. Der Ursprung dieser senkrechten Bündel ist sicher in dem feinen Netzwerke zu suchen, das geht aus dem ganzen Verlaufe derselben deutlich hervor; einen directen Uebergang des einen in das andere wirklich gesehen habe ich indessen bis jetzt noch nicht. Sie endigen auf der anderen Seite, indem sie sich wieder horizontal umbiegen, sich pinselförmig zertheilen und dann auf die Gangliengruppen an den Vordersträngen und das zwischen denselben befindliche Netz zueilen, mit dem sie zum grössten Theile sich verbinden, um dann in die vorderen Wurzeln einzutreten. Es sind also wieder 'Treppenbündel, welche verschiedene Stockwerke des Rückenmarks miteinander in Verbindung setzen (vierte Abtheilung). Dieses ist die eine aus dem Netz entstehende Faserart. Zweitens finden wir noch rein horizontal verlaufende Bündel, welche sich aus dem Netzwerk bilden, und die verticalen Züge rechtwinklig schneidend, sich ebenso wie die aus den letztern sich umbiegenden Bündel pinselförmig zertheilen, um 
sich mit den Nachbarn, mögen dieses nun rein horizontale, oder aus verticalen entstandene sein, zu durchflechten; dann entstehen aus diesem Fasergemisch wieder neue Bündel, die nun also Fasern aus verschiedenen Höhen des Rückenmarks führen, diese treten zu den Ganglienzellenhäufchen der vorderen Gruppe, bilden theils das feine Netz daselbst, in dem sie sich nun wieder mit den von den Seitensträngen aus eintretenden Fasern vermischen würden, theils treten sie durch und billen so zusammen mit den aus diesem letzten Netze sich wieder sammelnden Bündeln die vorderen durchtretenden Wurzeln, deren Verlauf man an diesem Präparat auf das beste sieht. Die Zahl der einzelnen Bündel innerhalb der weissen Substanz, welche sich schliesslich zu einer Nervenwurzel vereinigen, ist übrigens auf der Zeichnung genau dieselbe, wie die auf dem Präparate, und also eine ziemlich beträchtliche, ebenso ist der steife radienartige Verlauf vollständig natürlich. Die vorderen durchtretenden Wurzeln stehen also mit den Fasern der Hinteıstränge derselben Rückenmarkshälfte auf mehrfache Weise in Verbindung:

1. Von den Hintersträngen laufen dicke Faserbündel (mit und ohne Netz, mit und ohne Höhenverschiebung) bis zu den Ganglien der vorderen Gruppe, dann Netz und austretende Bündel.

2. Von den Hintersträngen laufen Fasern (mit den vorigen Nebenbedingungen) $\mathrm{zu}$

a) der mittleren hinteren,

b) der seitlichen Gangliengruppe,

hier erstes Netz, aus diesem Netz dann weiter zu den Ganglien der vorderen Gruppe und hier dann entweder noch einmal Auflösen in das zweite Netz oder directer Durchtritt.

3. Von den Hintersträngen laufen Fasern (Nebenbedingungen wie oben) direct bis zu den vorderen Gangliengruppen, ziehen zwischen den Häufchen derselben hindurch, ohne mit dem Netz in Verbindung zu treten und gehen dann mit den vorderen Wurzeln weiter (fünfte Abtheilung).

Diese letzte wäre eine sehr merkwürdige Art der Verbindung, indessen haben mir die Präparate, besonders das unter 5 abgebildete die Existenz derselben wenigstens sehr wahrscheinlich gemacht.

Es bliebe uns nun noch die Betrachtung des Faserverlaufs in der weissen Substanz, doch ist derselbe, soweit ihn meine Präparate erkennen lassen, nothwendigerweise schon bei der Beschreibung mitberücksichtigt worden, so dass ich es nicht für nöthig halte, noch 
einmal näher darauf zurückzukommen; und nur einen Punkt, der bisher unerwähnt blieb, möchte ich noch berühren, ich meine die Entstehung und netzartige Verflechtung der aus den Hintersträngen hervorgehenden Nervenbündel in der weissen Substanz jener und den Eintritt der hinteren Wurzel in ihre Verbindung mit jenem Netz.

Fig. 7 welche, wie schon erwähnt, die hintere Partie der Querschnittshälfte 2, b bei stärkerer Vergrösserung (Oberhäusersche Zeichenkammer und Obj. 5) darstellt, zeigt uns diese Verhältnisse sehr klar. Wir sehen hier links das dicke Bündel der eintretenden hinteren Wurzeln, welches wiederum aus verschiedenen kleineren Bündel zusammengesetzt ist. Jedes dieser kleineren Bündel sendet sowohl direct nach vorn als auch nach der Mitte zu laufende Faserzüge $a b$, es müssen also vielfach Kreuzungen dieser Fasern stattfinden, zumal da auch die nach der Mitte zu verlaufenden nicht einmal parallel laufen, sondern oft convergiren. Diese Fasern nun endigen theils in der weissen Substanz, indem sie sich, wie dies ja bekannt ist, zu senkrecht verlaufenden Fasern umbiegen, theils treten sie direct mit jenem Faserbündelnetz in Verbindung, das oben bereits erwähnt wurde, theils treten einige dünne grade nach vorne gehende Bündel direct in die graue Substanz ein. Diese letzteren sind auf dem vorliegenden Präparate übrigens weniger deutlich als auf vielen anderen. Ausser diesen den Wurzeln angehörigen Bündeln finden wir also noch ein von dickeren und dünneren Nervenbündeln gebildetes Netz, welches die gesammte Substanz der weissen Hinterstränge durchzieht. Diese Nervenbündel werden einmal und zum bei Weitem grösseren Theil gebildet aus solchen Fasern, welche in den Hintersträngen eine Zeit lang senkrecht verlaufen sind und nun umbiegen, um in die graue Substanz einzutreten, und zweitens aus dem Theil der hinteren Wurzelfasern, welcher ebenfalls seinen Lauf nach der grauen Substanz nimmt. Wie in einem Gebirge von allen Seiten von den Felsabhängen kleine Wasseradern herab rieseln, um sich zu immer grösseren und grösseren Bächen in den Thälern zu vereinigen, so sehen wir hier von den verschiedensten Theilen der Hinterstränge her ganz feine Nervenbündelchen nach verschiedenen Punkten zusammenlaufen, um gemeinsam ihren Weg weiter fortzusetzen. Hier finden dann aber unterwegs zwischen benachbarten Zügen die mannichfachsten Verbindungen statt, wieder eine Vermischung der Fasern der verschiedensten Gegenden, so dass schliesslich, wenn nun die allmählig ziemlich dick gewordenen 
Bündel in die graue Substanz eintreten, die grossen und kleinen dann aus ihnen entstehenden Faserzüge, welche nach vorn weiter verlaufen, aus den verschiedensten Theilen der Hinterstränge Fasern erhalten, und nicht nur daher, sondern auch von der Peripherie des Körpers direct kommende, da ja ein Theil der Wurzelfasern in das Netz eintritt. Die in der grauen Substanz befindlichen Bündel kreuzen sich dann noch vielfach, wie schon oben besprochen, und so ist dann der schliessliche Erfolg der, dass sowohl zu den verschiedenen Ganglienzellengruppen als auch zu der vorderen und hinteren Commissur Fasern aus sehr vielen Theilen der Hinterstränge ihren Weg nehmen. Es enthält also durchaus nicht ein bestimmter Faserstrang der Hinterstränge nur Fasern, die nach ein em bestimmten Punkte der grauen Substanz verlaufen.

Die in die hinteren Wurzeln eintretenden Fasern laufen also einmal direct $z u$ den Bündeln der grauen Substanz hin, zweitens werden sie zu Fasern, die senkrecht in der weissen Substanz weiter verlaufen.

Die Nervenbündel, welche aus den Hintersträngen in die graue Substanz treten, erhalten einmal Fasem, welche direkt aus den hinteren Wurzeln zu ihnen durchtreten, zweitens solche, welche aus einem vertikalen Verlauf in den Hintersträngen in den horizontalen umbiegen.

Möge es mir endlich noch gestattet sein, ein Präparat zu beschreiben, welches sich allerdings nicht genau an die eben besprochenen anschliesst, indessen doch von Interesse ist, da es uns über den Ursprung eines Theils der vorderen Wurzelfasern Aufschluss giebt.

Das Präparat ist ein mit Goldchlorid behandelter Querschnitt aus der Gegend des vierten Halswirbels vom Hunde, und zeigt die Verhältnisse, welche die beiliegenden beiden Zeichnungen, die Herr cand. med. Killian für mich anzufertigen so gütig war, uns darstellen, auf das Klarste, so dass ein Zweifel nicht aufkommen konnte. Wir sehen auf Fig. 8 und 9 , welche dieselbe Stelle beì verschiedener Vergrösserung darstellen (Hartnack Obj. 5 und 9 à immers., beides verkleinert) den vorderen Theil des rechten grauen Vorderhorns mit den grossen daselbst befindlichen Ganglienzellen, und die verschiedenen Züge der durchtretenden Wurzeln mit den dazwischen in grosser Menge liegenden Nervenfaserquerschnitten, den sogenannten Sonnenbildchen, welche übrigens in der Zeichnung im Verhältniss zu den Ganglienzellen viel zu gross gemacht sind. 
In der Mitte des Bildes etwa erblicken wir ganz am Rande der grauen Substanz zwischen drei Blutgefässquerschnitten gelegen eine Ganglienzelle mit Kern und vier Ausläufern. Zwei dieser letzteren ziehen rückwärts in die graue Substanz hinein, ohne dass ich nachweisen konnte, was weiter aus ihnen wird, zwei andere dagegen treten in zwei durch die weissen Stränge hindurchtretende vordere Wurzeln ein und sind in diesen deutlich eine ziemliche Strecke weit zu verfolgen. Dieser auch von Herrn Prof. Wald eyer constatirte Befund lässt es also wohl nicht zweifelhaft erscheinen, dass wir es hier mit einer Ganglienzelle zu thun haben, von der aus zwei Fortsätze zu Nervenfasern werden, welche durch die vorderen Wurzeln austretend nach der Peripherie des Körpers verlaufen. Es würde dieses Präparat also eiumal zu der geringen Zahl derjenigen bisher beschriebenen hinzutreten, welche den Beweis liefern, dass wirklich ein directer Fortsatz einer Ganglienzelle zu einer austretenden Wurzelfaser werden kann, zweitens aber würde es beweisen, dass eine Nervenzelle nicht nur einen, sondern auch zwei solcher Fortsätze, d. h. also nicht nur einen, sondern auch zwei Deiters'sche Axencylinderfortsätze haben kann. Es ist dieses ein Befund, wie er meines Wissens aus dem Rückenmarke eines Säugethieres bisher noch nie beschrieben worden ist, und der an Interesse noch zunimmt, wenn man sich erinnert, dass vor etwa 25 Jahren schon $R$. Wagener in dem Centralorgane des Zitterrochens ebenfalls Ganglienzellen mit zwei Axencylinderfortsätzen auffand und beschrieb.

Werfen wir noch einmal einen Blick auf den Aufbau des Rückenmarks, wie er sich uns nach diesen Beobachtungen dargestellt hat, so finden wir, dass zunächst ein Princip dabei in der consequentesten Weise durchgeführt worden ist: das Princip der möglichst vielseitigen Verbindung. Um dasselbe nun auf möglichst einfache und elegante Weise durchzuführen, sind verschiedene Mittel angewandt worden. (Sit venia verbo!)

1. Verschiedener Austritt der Fasern aus der weissen Substanz in die graue.

a) Die Fasern, die von demselben Punkte ausgehen, können in verschiedenen Höhen in die graue Substanz eintreten.

b) Fasern, die von verschiedenen. Punkten herkommend in verschiedenen Theilen der weissen Substanz verlaufen, biegen an derselben Stelle in die graue Substanz ein.

c) Fasern, welche denselben senkrecht verlaufenden Bündeln an- 
gehören, und auch in derselben Höhe nach der grauen Sub$\operatorname{stanz}$ hin umbiegen, gehen oft schon während des horizontalen Verlaufs in der weissen Substanz nach rechts und links hin auseinander und treten so in die graue Substanz zwar in derselben Horizontalebene ein, aber doch in Bündel, welche ihrem Zielpunkte nach verschiedenwerthig sind.

2. Verschiedene Arten des Verlaufs der Fasern in der grauen Substanz zum Zwecke der Faservermischung.

a) Einfache Netze (ohne Ganglienzellen).

Diese können wiederum zweierlei Natur sein, je nachdem sie gleich am Rande der weissen Substanz liegend nur die einzelnen eben ausgetretenen Bündel unter sich verbinden - primäre Netze, oder weiter nach der Mitte der grauen Substanz zu liegend Bündel von Fasern, welche bereits ein Netz durchgemucht haben, in Verbindung setzen - secundäre Netze. Alle diese Netze denke ich mir indessen nur durch Verflechtung, nicht durch wirkliche Anastomosen entstanden.

b) Faserzüge, welche verschiedene grössere Theile des Rückenmarks verbinden; dahin gehören:

$\alpha)$ die hinteren und vorderen Commissurfasern zur Verbindung der in den beiden Rückenmarkshälften gelegenen Theile, und

$\beta$ ) die senkrecht in der grauen Substanz aufsteigenden, Punkte in verschiedenen Hühen des Rückenmarks miteinander verbindenden Treppenfasern.

3. Besondere sehr wahrscheinlich wenigstens zur Verbindung verschiedenartiger Fasern dienende nervöse Apparate: die Ganglienzellen mit den dichten zwischen ihnen befindlichen Netzen, Könnte man eine solche Gangliengruppe heil herausnehmen und besehen, würde sie wahrscheinlich mit ihren nach allen Richtungen hin ausgehenden Faserbündeln einem zusammengerollten Igel nicht unähnlich sehen.

4. Endlich gehört dahin auch noch die Art und Weise des Eintritts der Fasern der hinteren Wurzeln mit ihren Kreuzungen und ihrem weiteren horizontalen und verticalen Verlauf.

Bedenken wir nun endlich noch, dass oft mehrere, bis vier bis fünf dieser eben angeführten Verflechtungsarten hintereinander in den Lauf derselben Fasern eingeschaltet sind, so erhalten wir eine solche Menge von Verbindungen, eine solche Menge von Wegen für die Leitung zu den vershiedensten Theilen des Rückenmarks, wir 
haben ferner in der sehr bedeutenden Anzahl von Ganglienzellen so viele Uebertragungsapparate, denen wieder aus den verschiedensten Theilen Reize zugeführt werden, dass wir wohl auch bei den complicirtesten Thätigkeiten, die wir nach den bisherigen durch das physiologische Experiment gelieferten Thatsachen dem Rückenmark zuschreiben können, dasselbe als einen, allerdings sehr complicirten Reflexmechanismus anzusehen berechtigt sind.

Strassburg, 26. September 1873.

Erklärung der Abbildangen auf Taf. XXXII, XXXIII a. XXXIV.

Alle Figuren sind um $2 / 9$ der Grösse verkleinert, Figur 7 um die Hälfte.

Fig. 1 a,b. Hartnack Obj. 3. und Cam. luc. Querschnitt aus dem Lendenmark eines Hundes in der Gegend des Ursprungs des I. Sacralnervenpaares. Färbung mit Palladiumchlorür. Die Partien der weissen Substanz sind nicbt weiter ausgeführt, nur die noch in der grauen Substanz liegenden weissen Stränge sind dunkel schraffirt.

Die auf $1, b$ mit Zahlen versehenen Striche deuten die Richtung an, in der die Längsschnitte, die den Zahlen entsprechen, den Querschnitt schneiden würden.

V. Vorderstränge.

S. Seitenstränge.

H. Hinterstränge.

V.W. Fordere Wurzeln.

H. W. Hintere Wurzoln.

F.a. und F.p. = Fissura anter. und poster.

Cmm. a. und Comm. p. = Commissura ant. und post.

C. c. Canal. centralis.

V. G. Vordere

S. G. Seitliche

Ganglienzellengruppe.

H. M. G. Hintere mittlere

P. R. $=$ Processus reticulares. 
494 Dr. P. Schiefferdecker: Beiträge z. Kenntniss d. Faserverlaufs etc.

Fig. $2 \mathrm{a}, \mathrm{b}$ Längsschnitte.

Fig. 2. Frontalschnitt. Goldchloridpräparat. Alles übrige wie bei der vorhergehenden Figur.

a. Stück des das Rückenmark zunächst umgebenden Bindegewebes.

b. Seitenstrang.

c. Austretende Nervenbündel. (Siehe Text.)

d. Den Can. centr. umgebendes Bindegewebe.

e. Epithel des Canal centr.

Fig. 3. Sagittalschnitt. Alles Uebrige wie bei den vorhergehenden Figuren.

a. Seitenstrang.

b. Austretende Nervenbündel.

c. Seitliche Ganglienzellengruppe.

d. Vordere Ganglienzellengruppe.

e. Vorderstrang mit durchtretenden vorderen Wurzeln.

f. Bindegewebe.

$x^{\prime}$. Aufsteigende Nervenbündel.

Fig. 4. Wie die vorhergehende Figur.

Fig. 5. Wie die vorhergehende Figur.

a. mediale Partie des Hinterstranges.

$\Sigma^{\prime}$. aufsteigende Nervenbündel.

V.W. vordere Wurzeln.

Fig. 6. Wie die vorgehende Figur.
a. Seitenstrang.
b. austretende Nervenbündel.

Fig. 7. Hartnack. Obj. 5. Cam. luc. Theil von Fig. 1, b. stärker vergrössert.

Fig. 8 und 9. Theil eines Querschnitts aus dem Rückenmark des Hundes in der Gegend des Ursprungs des vierten Cervicalnervenpaares. Vorderhorn. Siehe Text. 